\section{Parasitosis intestinales en poblaciones Mbyá-Guaraní de la Provincia de Misiones, Argentina: aspectos epidemiológicos y nutricionales}

\author{
Intestinal parasitosis in Mbyá-Guaraní \\ populations from Misiones Province, Argentina: \\ epidemiological and nutritional aspects
}

Graciela Teresa Navone 1

María Inés Gamboa ${ }^{1}$

Evelia Edith Oyhenart 2

Alicia Bibiana Orden 2

\footnotetext{
1 Centro de Estudios Parasitológicos y de Vectores, Universidad Nacional de La Plata, La Plata, Argentina.

2 Centro de Investigaciones en Genética Básica y Aplicada, Universidad Nacional de La Plata. La Plata, Argentina.

Correspondencia G. T. Navone

Centro de Estudios Parasitológicos $y$ de Vectores, Universidad Nacional de La Plata. Calle 2, n. 584, La Plata (1900), Buenos Aires, Argentina. gnavone@cepave.edu.ar
}

\begin{abstract}
Intestinal parasite infestation in indigenous Mbyá-Guaraní communities in Misiones, Argentina, was described and associated with nutritional status and environmental and cultural factors. The results were compared with those from Takuapi, a neighboring indigenous population, and the nearest urban population, Aristóbulo del Valle. The Ritchie, Willis, and Kato Katz techniques were used to analyze the stool samples. Anthropometric parameters were analyzed and earth samples processed. From a total sample of 296 individuals analyzed in the four populations, 100 (87.7\%), 63 (88.7\%), 49 (96.1\%), and 50 (82\%) were infested in Kaaguy Poty, Yvy Pytá, Takuapí, and Aristóbulo del Valle, respectively. $84 \%$ of infested individuals had multiple parasites. The $43 \%$ of the individuals presented malnutrition, and $87 \%$ of these were infested. There was an association between use of latrines and Giardia lamblia $(p<0.01)$; open-air defecation, lack of footwear, and hookworms ( $p<0.01)$; and housing type and total helminthes $(p<0.01)$. Earth samples were contaminated with parasites. The results suggest the relationship between environmental contamination and high prevalence of intestinal parasites in these human populations.
\end{abstract}

Helminths; Nutritional Status; South American Indians

\section{Introducción}

Las parasitosis intestinales y la contaminación fecal representan un importante problema que enfrenta la salud pública y ambiental en los países en vías de desarrollo 1,2. Estas infecciones son generalmente subestimadas por ser asintomáticas, pero sus efectos pueden contribuir a la morbilidad cuando están asociados a la malnutrición ${ }^{1}$. En la mayoría de los casos se cuenta con datos de prevalencia fragmentarios, procedentes de niños en edad escolar o de hospitales, que no son representativos del total de la población en un área determinada 2 . Si bien existen estudios de campo desarrollados en diferentes poblaciones, no han sido utilizados en la elaboración de medidas preventivas, ni han sido devueltos a las poblaciones involucradas, de manera que participen en la búsqueda de alternativas para la protección de la salud 1,2 .

El efecto del cambio cultural y la degradación ambiental en la prevalencia de infecciones parasitarias ha sido estudiado en poblaciones indígenas americanas 3,4 . Fitton 4 sostuvo que los procesos de colonización y de explotación de los recursos naturales han contribuido a la progresiva aculturación de poblaciones indígenas de Ecuador. Esta situación, unida al sedentarismo y la deficiente atención de la salud, favoreció la transmisión de infecciones parasitarias en esas poblaciones 3,4 . Por otra parte, también las infecciones parasitarias causan o agra- 
van la malnutrición y provocan un retraso en el crecimiento de los individuos infectados 5,6,7,8.

Desde un punto de vista ecoepidemiológico, la contaminación del suelo y el agua, las prácticas de defecación, los patrones de higiene y el hacinamiento representan los factores que más influyen sobre la prevalencia de las parasitosis intestinales 2,9.

Las comunidades Mbyá-Guaraní llegaron al territorio argentino procedentes del Paraguay en las primeras décadas del siglo XX, conformando los primeros asentamientos en las proximidades de las rutas 12 y 14 de la provincia de Misiones, Argentina 10,11. Dos de estas comunidades están asentadas en la Reserva Privada "Valle del Arroyo Cuña-Pirú" de la Universidad Nacional de La Plata (UNLP). Estudios etnográficos realizados en estas comunidades permitieron conocer, desde una perspectiva local, cuales son las enfermedades que los afectan y las estrategias utilizadas para enfrentarlas. Algunos de los síntomas de infección parasitaria reconocidos se refieren a la diarrea, color pálido o amarillo de la piel, deshidratación del globo ocular, dolores abdominales y retraso en el crecimiento, entre los más frecuentes 12 . Esta situación, unida a las características ambientales del área y el estilo de vida de los integrantes de estas poblaciones 13 , condujo a plantear el presente trabajo. El objetivo fue evaluar las enteroparasitosis y el estado nutricional de las poblaciones infantil y adulta de Kaaguy Poty e Yvy Pytá, observando los factores socio-ambientales que contribuyen a la transmisión parasitaria. Se propuso también comparar la epidemiología de las enteroparasitosis con otra comunidad Mbyá-Guaraní (Takuapí), ubicada fuera del ámbito de la reserva y con la población periurbana más cercana, Aristóbulo del Valle.

\section{Material y métodos}

La provincia de Misiones es la eco-región con mayor biodiversidad de Argentina 14 y se ha transformado en uno de los ecosistemas prioritarios a nivel mundial, desde una perspectiva científica y política.

Las comunidades Kaaguy Poty e Yvy Pytá habitan parte de las tierras declaradas Reserva Privada "Valle del Arroyo Cuña-Pirú" de la UNLP y mantienen contacto variable con la población urbana más cercana, Aristóbulo del Valle. Están asentadas al nordeste de la República Argentina, en el centro de la provincia de Misiones (5455'09"W y 5501'34"W; 2708'54"s y $27^{\circ} 03^{\prime} 55^{\prime \prime}$ ). La reserva tiene una superficie de 6.035ha, de las cuales 5.405 hectáreas (90\%) cor- responden al Departamento Cainguas, Municipio Aristóbulo del Valle y 630 (10\%) ha al Departamento Libertador General San Martín, Municipio de Garuhapé (Plano de Mensura N. 15.875 de la Dirección General de Catastro de la Provincia de Misiones. Convenio UNLP - Ministerio de Ecología) (Figura 1).

La reserva "Valle del Arroyo Cuña-Pirú" (Decreto n. 841, 30/Junio/2000) es atravesada en su parte norte por la Ruta Provincial № 7 y su límite nordeste conecta con las primeras urbanizaciones de Aristóbulo del Valle. Pertenece a dos formaciones fitogeográficas, el Distrito de las Selvas Mixtas y el Distrito de los Campos, ambos dentro de la eco-región Selva Atlántica. Constituye una de las unidades de conservación más importantes de Selva Atlántica Interior, situada en la terminal más austral de las selvas continuas de Misiones y del Corredor Verde 14. La Selva Atlántica Interior se denomina en Argentina Selva Paranaense o Misionera y se extiende a lo largo de la costa oriental de Brasil, este de Paraguay y nordeste de Argentina 15. El clima es cálido y húmedo con una temperatura media anual de $20^{\circ} \mathrm{C}$ y precipitaciones de entre 1.500 y $2.000 \mathrm{~mm}$ anuales. El relieve es fuertemente ondulado con colinas y llega a una altura de $475 \mathrm{~m}$ en Aristóbulo del Valle, la ciudad más cercana, distante a $12 \mathrm{~km}$. Los suelos son profundos areno-arcillosos, de textura gruesa que dificulta la evaporación. La cobertura vegetal esta formada por 4 ó 5 estratos verticales, con flora y fauna muy abundantes. Las comunidades Mbyá-Guaraní asentadas en el territorio misionero comprenden 700 familias (3.500 personas). Kaaguy Poty e Yvy Pytá están distribuidas en viviendas asociadas a espacios de cultivo, en claros abiertos de la selva a la vera de la Ruta Provincial n. 7. Según censo realizado en el 2003, la población total es de 285, correspondiendo 171 personas que ocupan 24 viviendas en Kaaguy Poty y 114 en 18 viviendas en Yvy Pytá. El promedio de hijos es de cuatro por familia nuclear 16. Los desplazamientos individuales o de grupos familiares imprimen a estos asentamientos una dinámica particular. Es una población joven, con una distribución de $77,2 \%$ menor de 30 años y 5,6\% mayor de 60 años 16. Las actividades de subsistencia incluyen la horticultura, caza, pesca, recolección, venta de artesanías y trabajos temporales en las plantaciones de yerba mate 16,17.

Kaaguy Poty e Yvy Pytá cuentan con una sala de primeros auxilios a cargo de un agente sanitario aborigen y ocasionalmente es visitada por un profesional médico. Dichas comunidades se encuentran a $12-15 \mathrm{~km}$ de distancia del hospital público de Aristóbulo del Valle. Los 

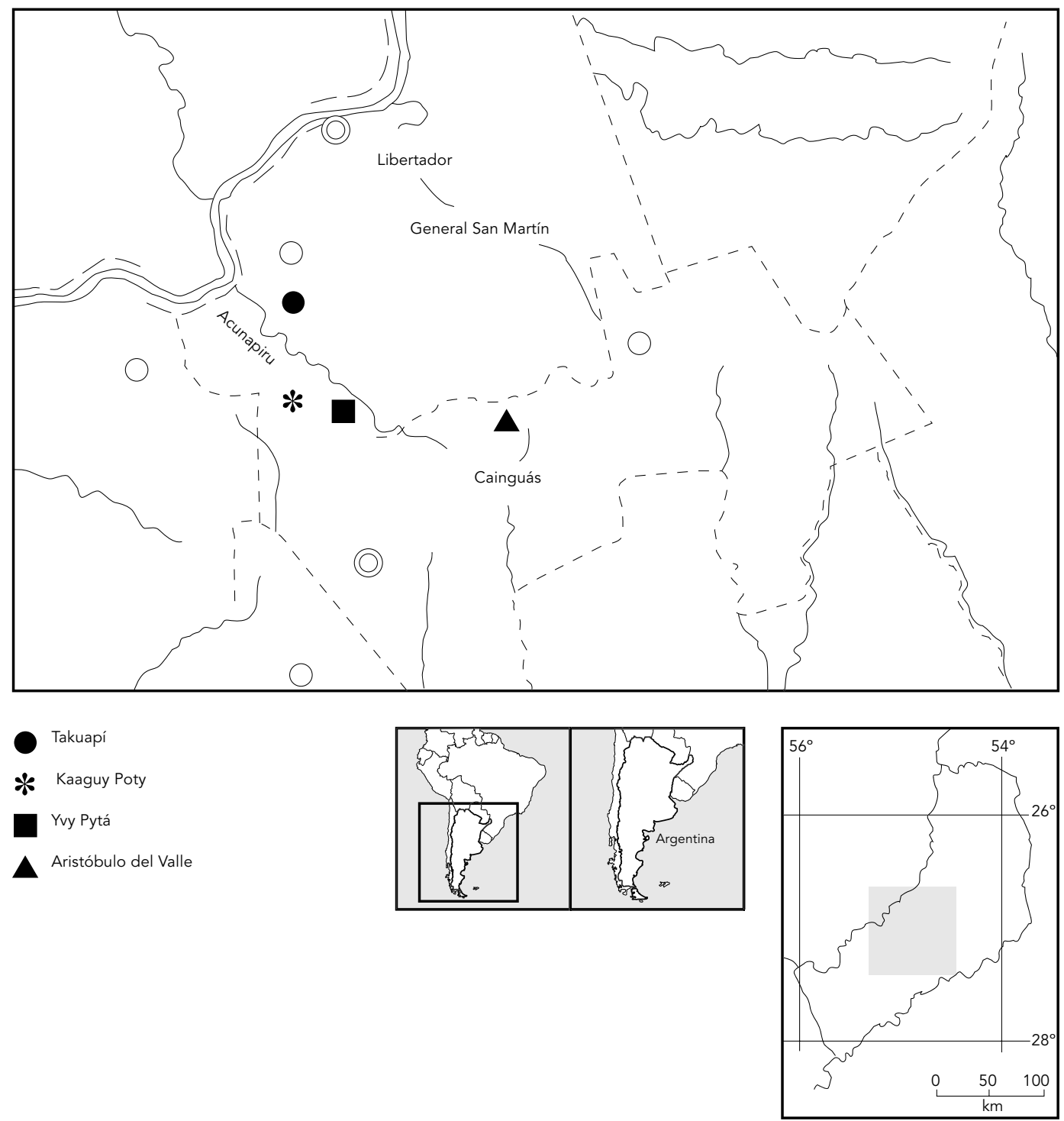

miembros de estas comunidades conocen al "Kaá-re" (Chenopodium anthielminticum) y "marcela" (Achyrochline satureioides) para el tratamiento de las parasitosis y otras dolencias, sin intervención de especialistas 12. Takuapí es otra comunidad Mbyá-Guaraní, asentada en el Partido de Ruiz de Montoya que dista $40 \mathrm{~km}$ de Aristóbulo del Valle, integrada por 80 individuos aproximadamente. Las tres poblaciones aborígenes ocupan viviendas de construcción precaria, hechas en madera y/o material, con pisos de tierra y / o cemento. La defecación es a cielo abierto y el agua de consumo proviene del arroyo Cuña-Pirú y de pozo (en pocos casos), que siempre conservan en recipientes por varios días debido a la distancia entre sus viviendas y las fuentes de obtención. La población de Aristóbulo del Valle está compuesta principalmente por descendientes de inmigrantes europeos y según censo de 1990 cuenta con 3.294 habitantes que ocupan 1.626 viviendas. La población relevada en esta localidad se asienta en 
el ámbito periurbano y sus viviendas son de material, con piso de cemento. El agua de consumo proviene de pozo y como método de eliminación de las excretas usan letrinas.

El presente estudio fue evaluado y aprobado en sus distintos aspectos, incluyendo los bioéticos, por la UNLP. Todos los niños y adultos evaluados consintieron participar en el estudio por escrito o verbalmente. Las investigaciones llevadas a cabo sobre las personas se ajustaron a lo establecido por la Declaración Universal de los Derechos Humanos de 1948, las normas éticas instituidas por el Código de Nüremberg de 1947 y la Declaración de Helsinki de 1964 y sucesivas enmiendas, atendiéndose especialmente a lo normado por la Ley Nacional n.25.326 de protección de datos personales.

\section{Relevamiento parasitológico}

Se tomaron 296 muestras fecales correspondientes, en la mayoría de los casos, a una sola deposición debido a la dificultad de obtener muestras seriadas en las poblaciones analizadas (113 de Kaaguy Poty, 71 de Yvy Pytá, 51 de Takuapí y 61 de Aristóbulo del Valle). La media de edad del total de la población relevada fue 10,5 años, y el rango fue de 10 meses a 82 años. Las muestras se conservaron en formol al $10 \%$, y para el análisis coproparasitológico se utilizaron técnicas de concentración por sedimentación (Ritchie, $\delta=1010$ ) y por flotación (Willis, $\delta=1200$ ) 18. Las cargas parasitarias fueron estimadas mediante la técnica de Kato Katz 19. Para el análisis parasitológico de las muestras de suelo se tomaron muestras del entorno de las viviendas y escuelas de Kaaguy Poty e Yvy Pytá. Se ensayó una técnica que utilizó $50 \mathrm{~g}$ de tierra en una dilución de $100 \mathrm{ml}$ de agua corriente y $2 \mathrm{ml}$ de solución de Tween 80 . La muestra se homogeneizó mediante un vibrador por un lapso de 45 minutos y por un sistema de 2 tamices de 140 micras de apertura el primero y 75 micras el segundo, la muestra se filtró mediante agua a presión. Se recogieron $750 \mathrm{ml}$ del filtrado, se dejó sedimentar por un período mínimo de 48 horas hasta observar un sobrenadante límpido, el cual fue eliminado por perforación del recipiente contenedor, cuidando conservar el doble de volumen del sedimento. El contenido fue resuspendido y se tomaron dos alícuotas en tubos de centrífuga. El rechazo del tamiz de 75 micras de apertura fue dividido y centrifugado durante cinco minutos a 3.000rpm, para luego desechar el sobrenadante. A un tubo se le agregó una solución de sacarosa de densidad igual a $1260 \mathrm{y}$ al otro, una solución de nitrato de sodio de densidad igual a
1290. Una vez resuspendido el sedimento, el material se centrifugó nuevamente a 3.000rpm por un lapso de 15 minutos y se tomaron muestras de la superficie para su prospección al microscopio óptico. Completada la primera revisión, el sobrenadante remanente de cada muestra se traspasó a nuevos tubos de centrífuga y se le agregó igual volumen de agua destilada. Previo centrifugado a 2.500rpm por cinco minutos, se pasó a un segundo análisis microscópico tomándose muestras del fondo.

\section{Análisis estadístico de los datos parasitológicos}

Se aplicó el índice de Fager 20 para medir la afinidad entre pares de especies parásitas que coocurrieron: $\mathrm{I}_{\mathrm{AB}}=2 \mathrm{j} / \mathrm{N}_{\mathrm{A}}+\mathrm{N}_{\mathrm{B}}$ donde $\mathrm{j}$ es el número de hospederos en los que las especies parásitas A y B están presentes, $\mathrm{N}_{\mathrm{A}}$ es el número de hospederos donde la especie A está presente y $\mathrm{N}_{\mathrm{B}}$ es el número de hospederos donde la especie B está presente. Para determinar si el índice de afinidad es estadísticamente significativo, se utilizó el test de t.

El coeficiente de similaridad de Sorensen 20 fue utilizado para expresar en términos porcentuales el grado de semejanza de las parasitosis observadas en cada comunidad examinada, $\mathrm{C}_{\mathrm{SS}}=2 \mathrm{C} / \mathrm{S}_{1}+\mathrm{S}_{2}$ donde $\mathrm{C}_{\mathrm{SS}}$ es el coeficiente de similaridad, $C$ número de especies comunes a ambas comunidades, $\mathrm{S}_{1}$ y $\mathrm{S}_{2}$ es el número de especies presentes (riqueza específica) en la comunidad $1\left(\mathrm{~S}_{1}\right)$ y en la comunidad $2\left(\mathrm{~S}_{2}\right)$.

\section{Relevamiento antropométrico}

Para la evaluación del estado nutricional se midieron un total de 107 individuos de ambos sexos de 2 a 60 años de edad en las poblaciones Kaaguy Poty e Yvy Pytá (Tabla 1). La edad de cada persona fue obtenida de su documento de identidad.

El estudio se realizó de acuerdo a protocolos estandarizados 21. El peso corporal se midió en kilogramos empleando una balanza digital portátil (10g de precisión) que se calibró al inicio de cada sesión. En todos los casos los individuos vistieron ropa liviana cuyo peso se descontó del peso total. La talla se midió en centímetros utilizando un antropómetro vertical (1mm de precisión), con el individuo descalzo y orientando la cabeza en el plano de Frankfort. Para la determinación del error intraobservador cada medición se realizó dos veces. La concordancia entre ambas mediciones fue evaluada mediante el coeficiente de correlación intraclase (CCI). Los valores de CCI superiores a 0,75 se consideraron aceptables. 
Estadística descriptiva para peso corporal y estatura en individuos Mbyá-Guaraní de Kaaguy Poty e Yvy Pytá.

\begin{tabular}{|c|c|c|c|c|c|c|c|}
\hline \multirow[t]{2}{*}{ Edad (años) } & \multirow[t]{2}{*}{$\mathbf{N}$} & \multicolumn{3}{|c|}{ Peso corporal $(\mathrm{kg})$} & \multicolumn{3}{|c|}{ Estatura $(\mathrm{cm})$} \\
\hline & & Media & $\begin{array}{l}\text { Desvío } \\
\text { estándar }\end{array}$ & Puntaje Z & Media & $\begin{array}{l}\text { Desvío } \\
\text { estándar }\end{array}$ & Puntaje Z \\
\hline $2,0-2,99$ & 14 & 11,6 & 2,2 & $-1,1$ & 81,6 & 5,4 & $-2,3$ \\
\hline $3,0-3,99$ & 4 & 14,2 & 2,6 & $-0,5$ & 94,6 & 5,0 & $-0,7$ \\
\hline $4,0-4,99$ & 5 & 15,3 & 2,0 & $-0,9$ & 97,5 & 6,2 & $-1,6$ \\
\hline $5,0-5,99$ & 7 & 16,6 & 1,7 & $-0,9$ & 100,5 & 4,7 & $-2,3$ \\
\hline $6,0-6,9$ & 7 & 19,2 & 2,7 & $-0,7$ & 110,3 & 7,0 & $-1,5$ \\
\hline $7,0-7,99$ & 15 & 21,2 & 2,8 & $-0,9$ & 115,7 & 4,8 & $-1,8$ \\
\hline $8,0-8,99$ & 5 & 24,3 & 2,7 & $-0,5$ & 119,9 & 4,6 & $-1,6$ \\
\hline $9,0-9,99$ & 13 & 26,4 & 5,2 & $-0,7$ & 125,4 & 10,0 & $-1,6$ \\
\hline $10,0-10,99$ & 9 & 28,5 & 3,5 & $-0,8$ & 130,5 & 7,4 & $-2,3$ \\
\hline $11,0-11,99$ & 8 & 30,2 & 5,3 & $-1,0$ & 131,6 & 6,0 & $-1,4$ \\
\hline $12,0-19,99$ & 6 & 39,2 & 6,7 & $-0,7$ & 140,2 & 5,9 & $-2,2$ \\
\hline $20,0-59,9$ & 14 & 52,7 & 5,6 & $-1,1$ & 152,2 & 7,2 & $-2,1$ \\
\hline
\end{tabular}

Todos los datos fueron transformados en puntaje Z, usando las referencias NHANES I y NHANES II 22. Para la determinación de prevalencias de retraso del crecimiento (baja talla/ edad o stunting), bajo peso/talla (wasting) y bajo peso/edad (underweight), se empleó el punto de corte de -2 DE por debajo de la mediana de referencia 23 .

\section{Relevamiento socioambiental}

El registro de los factores socioambientales se efectuó mediante observaciones sistemáticas, que permitieron conocer las condiciones y conductas que podrían ser indicadoras del riesgo de contraer infecciones parasitarias. Las características ambientales y culturales se asociaron estadísticamente a la presencia de las diferentes especies parasitarias. Se evaluaron el uso de calzado, los materiales y el piso de las viviendas y el sistema de eliminación de las excretas ${ }^{9}$. El análisis estadístico de estas variables en las cuatro comunidades relevadas se realizó mediante el programa Epi Info 6 (Centers for Disease Control and Prevention, Atlanta, Estados Unidos).

\section{Resultados}

Los quistes de amebas entéricas con cuatro núcleos y diámetro entre $12-15 \mu$ fueron identificados como Entamoeba sp., porque no se realizaron pruebas serológicas para diferenciar $E$. histolytica de E. dispar.
El número de personas estudiadas antropo/parasitológicamente coincidió parcialmente, ya que en el momento de la evaluación antropométrica, algunas familias se encontraban trabajando en las colonias, como personal temporal para la cosecha de yerba mate.

La prevalencia total de comensales y parásitos intestinales (protistas + helmintos) fue de 87,7\% en Kaaguy Poty, 88,7\% en Yvy Pytá, 96,1\% en Takuapí y $82 \%$ en Aristóbulo del Valle, observándose un total de 17 especies entre las cuales diez fueron protistas y siete helmintos.

La prevalencia observada en la población de Takuapí tuvo diferencias significativas sólo con Aristóbulo del Valle ( $\chi^{2}$ correcc. Maentel Haenszel $=5,34 ; p<0,05)$. La Tabla 2 muestra los valores de prevalencia para cada especie parásita en las cuatro poblaciones, diferenciando entre individuos menores y mayores de 14 años. Los ancilostomídeos fueron los parásitos más comunes en las tres poblaciones aborígenes y presentaron la menor prevalencia en la población periurbana de Aristóbulo del Valle $\left(\chi^{2}=74,11 ; \mathrm{p}<0,01\right)$. Por el contrario, Giardia lamblia fue mas frecuente en Aristóbulo del Valle que en las poblaciones aborígenes $\left(\chi^{2}=\right.$ 15,69; $\mathrm{p}<0,01)$. Iodamoeba butschlii, Hymenolepis nana y Ascaris lumbricoides fueron más frecuentes en las poblaciones aborígenes que en Aristóbulo del Valle $(\mathrm{p}<0,05)$.

La frecuencia de individuos mono y biparasitados fue mayor en Aristóbulo del Valle que en las poblaciones aborígenes, mientras que la frecuencia de poliparasitados fue superior al $60 \%$ en las poblaciones Mbyá y de $24 \%$ en Aris- 
Prevalencias (\%) parasitarias en las comunidades Mbyá-Guaraní y periurbana de Aristóbulo del Valle, distribuidas en dos rangos: 0-14 años y > 14 años.

\begin{tabular}{|c|c|c|c|c|c|c|c|c|}
\hline \multirow[t]{3}{*}{ Especie } & \multicolumn{4}{|c|}{$0-14$ años } & \multicolumn{4}{|c|}{$>14$ años } \\
\hline & Kaaguy Poty & Yvy Pytá & Takuapí & $\begin{array}{l}\text { Aristóbulo } \\
\text { del Valle }\end{array}$ & Kaaguy Poty & Yvy Pytá & Takuapí & $\begin{array}{c}\text { Aristóbulo } \\
\text { del Valle }\end{array}$ \\
\hline & $n=90$ & $n=61$ & $n=36$ & $\mathrm{n}=59$ & $\mathrm{n}=24$ & $n=10$ & $\mathrm{n}=15$ & $n=2$ \\
\hline Protozoos & $65(72,2)$ & $44(72,1)$ & $30(83,3)$ & $41(69,5)$ & $21(87,5)$ & $9(90,0)$ & $14(93,3)$ & $1(50,0)$ \\
\hline G. lamblia & $11(12,2)$ & $7(11,5)$ & $5(13,9)$ & $20(34)$ & $3(12,5)$ & $1(10,0)$ & $10(66,7)$ & 0 \\
\hline E. coli & $46(51,1)$ & $28(45,9)$ & $17(47,2)$ & $18(30,5)$ & $20(83,3)$ & $7(70,0)$ & $10(66,7)$ & $1(50,0)$ \\
\hline B. hominis & $28(31,1)$ & $26(42,6)$ & $24(66,7)$ & $18(30,5)$ & $7(29,2)$ & $2(20,0)$ & $1(6,7)$ & 0 \\
\hline E. nana & $16(17,8)$ & $13(21,3)$ & $3(8,3)$ & $7(11,9)$ & $8(33,3)$ & $1(10,0)$ & $4(26,7)$ & 0 \\
\hline I. butschlii & $13(14,4)$ & $7(11,5)$ & $4(11,1)$ & $3(5,1)$ & $5(20,8)$ & $2(29,0)$ & $1(6,7)$ & 0 \\
\hline Entamoeba sp. & $3(3,3)$ & $3(4,9)$ & 0 & $1(1,7)$ & $2(8,3)$ & 0 & 0 & 0 \\
\hline C. parvum & $4(4,6)$ & 0 & 0 & 0 & $1(4,2)$ & 0 & 0 & 0 \\
\hline Cyclospora sp. & $2(2,3)$ & 0 & 0 & $1(1,7)$ & $3(12,5)$ & 0 & 0 & 0 \\
\hline E. hominis & 0 & 0 & 0 & $1(1,7)$ & 0 & 0 & 0 & 0 \\
\hline Ch. mesnili & 0 & $3(4,9)$ & 0 & 0 & $1(4,2)$ & 0 & $1(6,7)$ & 0 \\
\hline Helmintos & $73(81,1)$ & $43(70,5)$ & $35(97,2)$ & $29(49,2)$ & $20(83,3)$ & $9(90,0$ & $15(100,0)$ & $1(50,0)$ \\
\hline Ancilostomídeos & $66(73,3)$ & $38(62,3)$ & $32(88,9)$ & $11(18,6)$ & $20(83,3)$ & $9(90,0)$ & $14(93,3)$ & $1(50,0)$ \\
\hline A. lumbricoides & $11(12,2)$ & $11(18)$ & $14(38,9)$ & $2(3,4)$ & $3(12,5)$ & 0 & $5(33,3)$ & 0 \\
\hline S. stercoralis & $19(21,1)$ & $16(26,2)$ & $20(55,6)$ & $20(34)$ & $3(12,5)$ & $3(30,0)$ & $4(26,7)$ & 0 \\
\hline H. nana & $20(22,2)$ & $8(13,1)$ & $8(22,2)$ & $2(3,4)$ & $6(25)$ & $1(10,0)$ & $7(46,7)$ & 0 \\
\hline E. vermicularis & $1(1,1)$ & 0 & $4(11,1)$ & $1(1,7)$ & 0 & 0 & 0 & 0 \\
\hline T. trichiura & $2(2,2)$ & $2(3,3)$ & 0 & $1(1,7)$ & $2(8,3)$ & 0 & $1(6,7)$ & 0 \\
\hline
\end{tabular}

tóbulo del Valle (Figura 2). Entre los monoparasitados, los ancilostomídeos fueron más frecuentes en los Mbyá $(74,4 \%)$ y G. lamblia, E. co$l i$ y $S$. stercoralis en la población periurbana $(32,8 \%)$. Entre los biparasitados, la asociación más frecuente fue ancilostomídeos y E. coli; en las asociaciones de tres especies la más frecuente fue entre ancilostomídeos, E. coli y $S$. stercoralis.

Las poliparasitosis estuvieron representadas en un $86 \%$ de casos entre los aborígenes, con mínimo de tres especies y un máximo de siete, siendo las especies más comunes $G$. lamblia, E. coli, B. hominis, H. nana, A. lumbricoides, ancilostomídeos y $S$. stercoralis. En la población periurbana, sólo en un caso se observaron seis especies (E. coli, B. hominis, Entamoeba sp., I. butschlii, Cyclospora sp. y E. nana).

Hubo asociación entre la edad y el sexo del hospedero, con la prevalencia de parásitos intestinales en Kaaguy Poty, evidenciándose que los más parasitados fueron los mayores de 14 años $\left(\chi^{2}=6,2, p<0,05\right)$ y los varones en el total de individuos parasitados $\left(\chi^{2}\right.$ correcc. Maentel Haenszel $=5,62 ; p=0,01)$. No hubo asociación entre el sexo y la edad de los hospederos con el número de especies parasitarias presentes.

Las cargas parasitarias se evaluaron en Kaaguy Poty e Yvy Pytá y las correspondientes a los geohelmintos (ancilostomideos, S. stercoralis, $A$. lumbricoides y T. trichiura) fueron leves en altos porcentajes (Tabla 3). La mayor afinidad se observó entre ancilostomídeos y B. hominis en Takuapí $(\mathrm{I}=0,82, \mathrm{t}=5,8)$, seguida de ancilostomídeos y E. coli $(\mathrm{I}=0,75, \mathrm{t}=6,27)$ en Kaaguy Poty. El resto de los pares de especies estudiadas en las poblaciones aborígenes y en la población periurbana presentaron menores valores del índice de Fager, tales como B. hominis-ancilostomídeos (poblaciones aborígenes: $\mathrm{I}=0,61, \mathrm{t}=5,64$; Aristóbulo del Valle: $\mathrm{I}=0,13, \mathrm{t}=4,25$ ) y $B$. hominis-S. stercoralis (poblaciones aborígenes: $\mathrm{I}=$ $0,42, \mathrm{t}=1,78$; Aristóbulo del Valle: $\mathrm{I}=0,16, \mathrm{t}=$ 4,50 ), entre las especies parásitas más comunes.

Los valores del coeficiente de similaridad (Sorensen) variaron entre 0,84 (84\%) y 0,91 (91\%), comprobándose que existe una elevada semejanza entre los resultados parasitológicos obtenidos en las cuatro poblaciones estudiadas.

De las 67 muestras de tierra analizadas en Kaaguy Poty e Yvy Pytá, 28 (41,8\%) fueron posi- 
Distribución de individuos mono y poliparasitados en las comunidades Mbyá-Guaraní y periurbana de Aristóbulo del Valle. Misiones, Argentina.

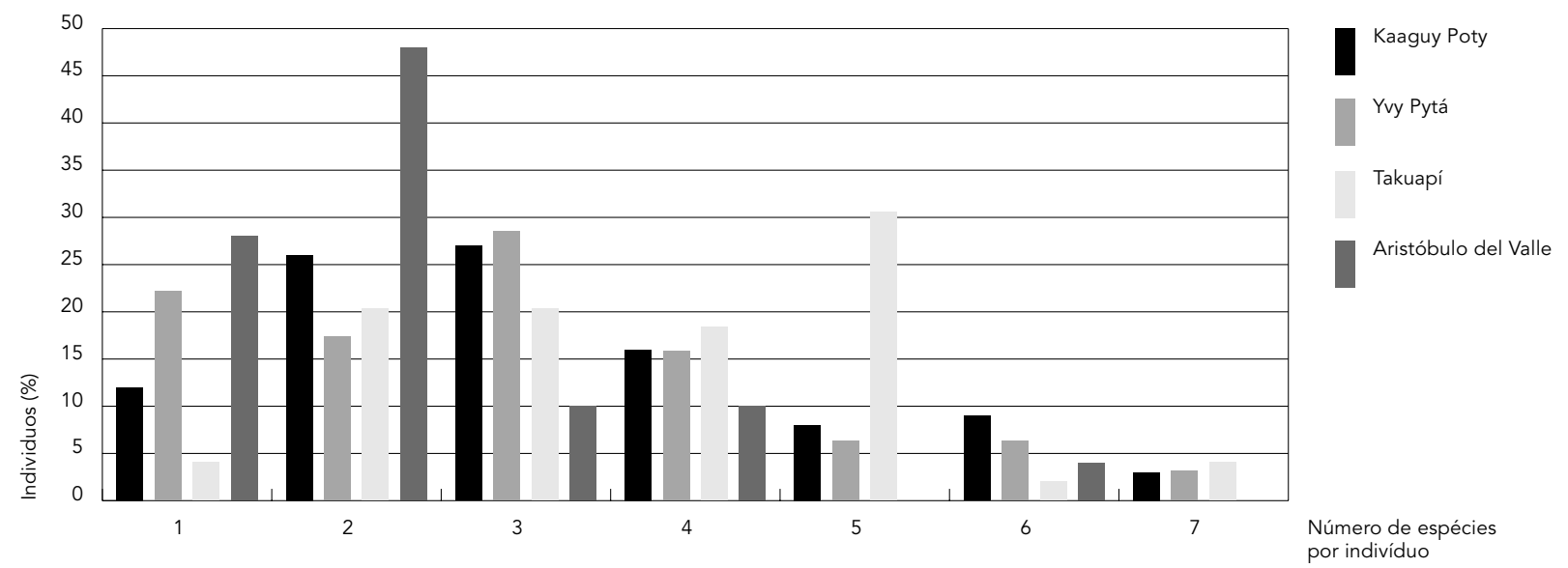

Tabla 3

Distribución de las cargas parasitarias en las comunidades de Kaaguy Poty e Yvy Pytá.

\begin{tabular}{lcccc}
\hline Parásito & Analizados (n) & $\begin{array}{c}\text { Infecciones } \\
\text { leves (\%) }\end{array}$ & $\begin{array}{c}\text { Infecciones } \\
\text { moderadas (\%) }\end{array}$ & $\begin{array}{c}\text { Infecciones } \\
\text { severas (\%) }\end{array}$ \\
\hline Ancilostomídeos & 104 & 75,0 & 8,7 & 17,0 \\
S. stercoralis & 29 & 93,1 & 0,0 & 6,9 \\
A. lumbricoides & 13 & 76,9 & 15,4 & 7,7 \\
T. trichiura & 4 & 100, & 0,0 & 0,0 \\
Total & 148 & 78,3 & 7,4 & 14,1 \\
\hline
\end{tabular}

tivas para alguna de estas especies: Trichuris sp., ancilostomídeos, Toxocara sp., Ascaris sp., $H$. nana y ooquistes de coccidios.

En la Tabla 1 figuran la estadística descriptiva y los valores $\mathrm{Z}$ medios de peso y estatura de la población evaluada parasitológica y antropométricamente $(\mathrm{N}=107)$. Como puede observarse los desvíos $\mathrm{Z}$ de peso y estatura fueron negativos en todas las edades, especialmente los correspondientes a la estatura, con valores cercanos o superiores a -2 . Hubo $43 \%$ (46) de individuos desnutridos, de los cuales $89,1 \%$ tuvo baja talla/edad, $4,4 \%$ bajo peso/edad y $6,5 \%$ bajo peso/talla. Del total de individuos desnutridos, 87\% (40) estaba parasitado, correspondiendo el mayor porcentaje a ancilostomídeos
$(67,4 \%)$. La asociación entre individuos desnutridos y parasitados no fue significativa $\left(\chi^{2}=\right.$ 0,$27 ; \mathrm{p}=0,6$ ).

La relación entre parasitosis/factores ambientales se presenta en la tabla 4 . Fue significativa la relación entre el piso de tierra de las viviendas y la presencia de ancilostomídeos $\left(\chi^{2}\right.$ correcc. Yates $=37,4 ; \mathrm{p}<0,01)$, de A. lumbricoides ( $\chi^{2}$ correcc. Yates $\left.=4,9 ; \mathrm{p}<0,05\right)$ y de $H$. $n a$ $n a\left(\chi^{2}\right.$ correcc. Yates $\left.=12,5 ; \mathrm{p}<0,01\right)$. Las viviendas construidas en madera estuvieron asociadas con la presencia de ancilostomídeos $\left(\chi^{2}\right.$ correcc. Yates $=38,30 ; p<0,01)$, de H. nana $\left(\chi^{2}\right.$ correcc. Yates $=12,73 ; \mathrm{p}<0,01)$, de A. lumbricoides $\left(\chi^{2}\right.$ correcc. Yates $\left.=5,02 ; \mathrm{p}<0,05\right), \mathrm{y}$ de helmintos en general $\left(\chi^{2}\right.$ correcc. Yates $=22,74$; 
Variables socioambientales asociadas estadísticamente a las infecciones parasitarias en las comunidades Mbyá-Guaraní y periurbana de Aristóbulo del Valle.

\begin{tabular}{|c|c|c|c|c|c|c|c|}
\hline \multirow[t]{2}{*}{ Variable } & \multicolumn{2}{|c|}{ Ancilostomídeos } & \multicolumn{2}{|c|}{ A. lumbricoides } & \multicolumn{2}{|c|}{ H. nana } & \multirow[t]{2}{*}{$p$} \\
\hline & Presencia & Ausencia & Presencia & Ausencia & Presencia & Ausencia & \\
\hline Piso & & & & & & & $<0,01$ \\
\hline Tierra $(n=165)$ & 80,0 & 20,0 & 20,0 & 80,0 & 24,8 & 75,1 & \\
\hline Cemento $(n=131)$ & 45,0 & 54,9 & 9,9 & 90,1 & 8,4 & 91,6 & \\
\hline Construcción & & & & & & & $<0,01$ \\
\hline Madera $(n=165)$ & 80,0 & 20,0 & 20,0 & 80,0 & 24,8 & 75,1 & \\
\hline Material $(n=132)$ & 44,7 & 55,3 & 9,8 & 90,1 & 8,3 & 91,6 & \\
\hline Calzado & & & & & & & $<0,01$ \\
\hline Ausente $(n=236)$ & 75,8 & 24,1 & 18,6 & 81,4 & 21,1 & 78,8 & \\
\hline Presente $(n=61)$ & 19,6 & 80,3 & 3,3 & 96,7 & 3,3 & 96,7 & \\
\hline Excretas & & & & & & & $<0,01$ \\
\hline Cielo abierto $(n=236)$ & 75,8 & 24,5 & 18,6 & 81,4 & 21,2 & 78,8 & \\
\hline Letrina $(n=61)$ & 80,3 & 19,6 & 3,3 & 96,7 & 3,3 & 96,7 & \\
\hline
\end{tabular}

$\mathrm{p}<0,01)$. El andar descalzo y la defecación a cielo abierto se asociaron a la presencia de ancilostomídeos, A. lumbricoides e H. nana $\left(\chi^{2}\right.$ correcc. Yates $=64,22 ; \mathrm{p}<0,01, \chi^{2}$ correcc. Yates = 7,$61 ; \mathrm{p}<0,01$ y $\chi^{2}$ correcc. Yates $=9,56 ; \mathrm{p}<0,01$ respectivamente). Las familias que usan letrina como forma de eliminación de excretas presentaron mayor prevalencia de G. lamblia $\left(\chi^{2}\right.$ correcc. Yates $=14,15 ; \mathrm{p}<0,01$ ).

\section{Discusión}

Las parasitosis intestinales son frecuentes en aquellos ambientes caracterizados por la pobreza, precariedad de las viviendas, prácticas sanitarias inadecuadas y hacinamiento 2,24,25. El presente estudio reveló elevadas prevalencias de parásitos intestinales, siendo los ancilostomídeos y E. coli los más frecuentes en las tres poblaciones aborígenes estudiadas y $G$. lamblia en la población periurbana. Si bien no se realizaron coprocultivos para determinar la identidad de los ancilostómideos, siguiendo el criterio de distribución de las especies patógenas humanas 26 y los síntomas reconocidos por estas poblaciones 12 se podría sugerir que $\mathrm{Ne}$ cator americanus es la especie que está presente en estas comunidades. Miranda et al. 24 observaron que $A$. lumbricoides y ancilostomídeos, fueron más prevalentes en tres aldeas indígenas de la Amazonia Oriental Brasilera.
Fontbonne et al. 25 en indígenas de Pernambuco indicaron que la especie más frecuente fue E. histolytica, seguida por A. lumbricoides y E.coli, mientras que los ancilostomídeos fueron menos comunes. Por otra parte Ferrari et al. 27 observaron que $A$. lumbricoides fue la especie más frecuente en el Estado de Rondonia, pero con un porcentaje inferior al $40 \%$. Las prevalencias parasitarias observadas en los aborígenes estudiados, se encuentran entre las más altas descritas para este tipo de poblaciones 28,29,30. Posiblemente estas diferencias se deban a la administración de tratamientos antihelmínticos a las poblaciones brasileñas, situación que no se observó en los Mbyá de Misiones. Ellos mencionaron al "Kaá-re" (C. anthielminticum), "marcela” (A. satureioides) y "verbena" (Verbena intemedia) entre los recursos vegetales más eficaces en las prácticas terapéuticas contra los parásitos 12 , pero la prevalencia se mantuvo elevada. Ello podría explicarse por la supervivencia y dispersión de las larvas, debido a la defecación a cielo abierto, al nivel de sombra y humedad en los sitios de defecación y a la alta pluviosidad del área. A estos factores se suma el hábito de andar descalzo y el desarrollo de actividades recreativas y laborales en contacto con el suelo, tanto en la población infantil como adulta 13. Sin embargo, aunque las prevalencias fueron altas (i.e., ancilostomideos), las cargas parasitarias fueron leves. 
Fitton ${ }^{4}$ y Ferrari et al. 27 consideraron que si bien existen poblaciones que permanecen aisladas, otras tienen mayor contacto con comunidades vecinas y ello favorecería la transmisión de las infecciones parasitarias. El quiebre de las creencias tradicionales en poblaciones aborígenes pudo llevar a deterioros en la salud y la degradación del agua de consumo, modificando estrategias de subsistencia y conduciendo a un aumento en la prevalencia de parasitosis intestinales. Esta situación caracteriza también a las comunidades Mbyá estudiadas, cuyo contacto con centros urbanos cercanos, podría relacionarse con alguna de las parasitosis observadas (i.e., G. lamblia).

En lo que respecta a la población periurbana de Aristóbulo del Valle, la elevada prevalencia parasitaria observada ( $82 \%)$ puede atribuirse a condiciones de extrema pobreza e inadecuada evacuación de las excretas mediante el uso de letrinas, que combinada con la extracción de agua de la napa freática contaminada, favorece la instalación y dispersión de las especies parásitas 2,9 .

La elevada prevalencia de ancilostomídeos y baja prevalencia de G. lamblia en los aborígenes contrasta con lo observado en la población periurbana, donde G. lamblia fue más común. Probablemente estas diferencias estén relacionadas con el uso de letrinas en un caso (Aristóbulo del Valle) y el andar descalzo y la defecación a cielo abierto en el otro (Kaaguy Poty; Yvy Pytá; Takuapí).

Al analizar la relación entre edad del hospedero, sexo y prevalencia total de parásitos intestinales, se observó una relación positiva sólo en Kaaguy Poty, donde los varones jóvenes estuvieron más parasitados, coincidiendo en parte, con los resultados obtenidos por Miranda et al. ${ }^{28}$ y Tsuyuoka et al. ${ }^{31}$ en Brasil. En lo que respecta a las parasitosis múltiples, los individuos de ambos sexos y de diferentes edades estuvieron en su mayoría poliparasitados en las tres poblaciones Mbyá, mientras que en Aristóbulo del Valle fueron superiores los valores de mono y biparasitismo. Esto puede asociarse tanto a las costumbres como a las condiciones del entorno de las poblaciones Mbyá, que favorecen la dispersión y las reinfecciones parasitarias.

El resultado del índice de Fager para la mayoría de los pares de especies indicó que la asociación entre ellas ocurrió al azar, a excepción de ancilostomídeos con B. hominis, ancilostomídeos con E. coli y B. hominis con S. stercoralis que fueron positivas en las poblaciones aborígenes, relacionado estrechamente al fecalismo ambiental. Los valores del coeficiente de
Sorensen demostraron que las mismas especies parásitas estuvieron presentes en un área con características ecológicas y socioculturales comunes.

El estudio parasitológico del suelo confirmó nuevamente que las parasitosis intestinales en estas poblaciones están asociadas a la contaminación del entorno 2,9. Las condiciones del suelo misionero (arcilloso, húmedo y con abundante materia orgánica), combinadas con las temperaturas elevadas, proveen un ambiente adecuado para el desarrollo y la supervivencia de huevos y larvas de helmintos. Niños y adultos desarrollan sus actividades en las viviendas y su entorno inmediato, que unido a la falta de uso de calzado facilitaría el transporte de quistes, huevos y larvas de parásitos hacia las viviendas, contaminando utensilios e indumentarias.

La malnutrición proteica en poblaciones transicionales, como las aquí estudiadas, ha sido asociada a altas cargas parasitarias 4 y la relación entre infecciones por geohelmintos, malnutrición y anemia ha sido demostrada por diversos autores 6,7,8,32,33. El elevado grado de parasitismo intestinal, junto a la presencia constante de otras enfermedades infecciosas como las respiratorias agudas, diarreas, etc., podrían contribuir al surgimiento de cuadros de anemia y/o malnutrición proteica en estas poblaciones. Del análisis de los parámetros antropométricos resultó que, en concordancia con lo observado previamente por Oyhenart et al. 34 , así como lo informado para otros grupos de Amerindios sudamericanos 35,36,37, la prevalencia de "stunting" fue significativamente más alta que el "underweight" y el "wasting". No obstante, la falta de asociación entre desnutrición y parasitosis, sumado a las bajas cargas parasitarias encontradas en el presente estudio, conducen a suponer que el estado nutricional de esta población resulta de la combinación de múltiples factores. Según Santos 38 las condiciones de vida en general son responsables del elevado porcentaje de desnutrición crónica. Al respecto, consideró que las carencias nutricionales específicas por la reducción de la diversidad de alimentos y las condiciones de precariedad sanitaria contribuyen a la cronicidad de la desnutrición. Aunque la subsistencia tradicional de los Mbyá fue variada combinando caza-recolección y eventualmente productos hortícolas (i.e., mandioca), los recursos alimentarios actuales son más escasos y menos variados que en tiempos históricos, con una reducida contribución de la proteína animal en la dieta diaria. Al respecto, Orr et al. 36 sugirieron que la calidad de la dieta (medida en pro- 
teínas animales) podría ser la causa de las mayores diferencias observadas en la estatura entre las poblaciones de Amazonia.

Los resultados sobre el estado nutricional, la creciente reducción del área que ocupan las comunidades aborígenes y la constante degradación del ambiente junto al sedentarismo y el incremento de la densidad poblacional, serían los factores que favorecen la transmisión y el aumento de la susceptibilidad a determinadas parasitosis (i.e., ancilostomídeos).

El patrón general de cambio ocurrido en los ecosistemas misioneros, asociado a cambios culturales, causó perjuicios en la salud humana y han sido similares a los procesos de aculturación observados en otras poblaciones 39 . El desarrollo de estudios que determinen la distribución relativa de las cargas parasitarias en las diferentes comunidades Mbyá de Misiones

\section{Resumen}

Se describieron las enteroparasitosis más frecuentes en dos comunidades Mbyá-Guaraní de Misiones, Argentina: Kaaguy Poty e Yvy Pytá. Las parasitosis intestinales se asociaron con el estado nutricional de los individuos infectados y los factores ambientales y culturales que caracterizan a las poblaciones estudiadas. Los resultados se compararon con los obtenidos en una población aborigen vecina, Takuapí, y la población urbana más cercana: Aristóbulo del Valle. Se utilizaron las técnicas de Ritchie, Willis y Kato Katz para las muestras fecales. Se analizaron los parámetros antropométricos y se procesaron muestras de tierra. De un total 296 individuos en las cuarto poblaciones, 100 (87,7\%), 63 (88,7\%), 49 (96,1\%) y 50 (82\%) estuvieron parasitados en Kaaguy Poty, Yvy Pytá, Takuapí, y Aristóbulo del Valle respectivamente. El $84 \%$ de los individuos parasitados estuvieron poliparasitados. El 43\% de los individuos medidos presentaron desnutrición y el $87 \%$ de ellos estaban parasitados. Hubo asociación entre el uso de letrinas $y$ Giardia lamblia $(p<0,01)$; defecación a cielo abierto, ausencia de calzado y ancilostomídeos $(p<0,01)$; el tipo de vivienda y los helmintos en general $(p<0,01)$. En el suelo se hallaron formas $p a-$ rasitarias, sugiriendo la relación entre la contaminación del entorno ambiental y la elevada prevalencia de parasitosis intestinales en las poblaciones estudiadas. podría ayudar a identificar individuos carriers, que debieran ser monitoreados y tratados periódicamente con el objeto de reducir el nivel de contaminación entre estas comunidades. Más aún, teniendo en cuenta que el impacto combinado de varias especies de parásitos intestinales es mayor que aquel producido por parásitos individuales 8 . La elevada frecuencia de poliparasitados observada, hace preocupante la situación de estas comunidades por el riesgo de cuadros graves, de procesos obstructivos y de formas invasivas, con sus consecuencias patológicas para la población afectada. Finalmente, los resultados obtenidos plantean la necesidad de profundizar los estudios integrando el saber científico y el aborigen, a fin de garantizar la eficacia de los programas de acción sanitaria.

\section{Colaboradores}

G. T. Navone fue responsable por el relevamiento de datos parasitológicos a campo, análisis de laboratorio y redacción del manuscrito. M. I. Gamboa contribuyó en el procesamiento estadístico y redacción del manuscrito. E. E. Oyhenart y A. B. Orden contribuyeron en el relevamiento, procesamiento de datos antropométricos y revisión del manuscrito.

\section{Agradecimientos}

Los autores agradecen a las autoridades de la Facultad de Ciencias Naturales y Museo de la Universidad Nacional de La Plata y del hospital municipal de Aristóbulo del Valle. A la Dra. Mampey de la Unidad Sanitaria de Takuapí; a la Dra. Crivos y su equipo de investigación y a todos los participantes del estudio. Agradecen también al editor y revisores anónimos por las sugerencias sobre el manuscrito.

Esta investigación ha sido parcialmente financiada por la Universidad Nacional de La Plata; el Consejo Nacional de Investigaciones Científicas y Técnicas (CONICET) y la Comisión de Investigaciones Científicas de la Provincia de Buenos Aires (CIC). 


\section{Referencias}

1. Carneiro F, Cifuentes E, Téllez-Rojo MM, Romieu I. The risk of Ascaris lumbricoides infection in children as an environmental health indicator to guide preventive activities in Caparaó and Alto Caparaó, Brazil. Bull World Health Organ 2003; 80:40-6.

2. Gamboa MI, Basualdo JA, Córdoba MA, Pezzani BC, Minvielle MC, Lahitte HB. Distribution of intestinal parasitoses in relation to environmental and sociocultural parameters in La Plata, Argentina. J Helminthol 2003; 77:15-20.

3. Confalonieri U, Ferreira LF, Araujo A. Intestinal helminths in Lowland South American Indians: some evolutionary interpretations. Hum Biol 1991; 63:863-73.

4. Fitton LJ. Helminthiasis and culture change among the Cofán of Ecuador. Am J Hum Biol 2000; 12:46577.

5. Stephenson LS, Latham MC, Ottesen EA. Malnutrition and parasitic helminth infections. Parasitology 2000; 121 Suppl:S23-38.

6. Stephenson LS, Latham MC, Kurz KM, Kinoti SN, Brigham H. Treatment with a single dose of Albendazole improves growth of Kenyan schoolchildren with hookworm, Trichuris trichiura and Ascaris lumbricoides infections. Am J Trop Med Hyg 1989; 41:78-87.

7. Brooker S, Peshu N, Warn PA, Mosobo M, Guyatt HL, Marsh K, et al. The epidemiology of hookworm infection and its contribution to anemia among pre-shool children on the Kenyan coast. Trans R Soc Trop Med Hyg 1999; 93:240-6.

8. Wilson WM, Dofour DL, Staten LK, Barac-Nieto M, Reina JC, Spurr GB. Gastrointestinal parasitic infection, anthropometrics, nutritional status and physical work capacity in colombian boys. Am J Hum Biol 1999; 11:763-71.

9. Gamboa MI, Basualdo-Farjat JA, Kozubsky L, Costas ME, Cueto-Rúa E, Lahitte HB. Prevalence of intestinal parasitosis within three population groups in La Plata, Buenos Aires, Argentina. Eur J Epidemiol 1998; 4:55-61.

10. Bartolomé MA. La situación de los Guaraníes (Mbyá) de Misiones (Argentina). Suplemento antropológico de la Revista del Ateneo Paraguayo 1969; 4:161-84.

11. Remorini C. Caminar a través del monte. Una aproximación a la movilidad Mbyá en el pasado y en el presente. In: Actas de las II Jornadas sobre Poblamiento, Colonización e Inmigración en Misiones. Posadas: Montoya Ediciones; 2001. p. 309-26.

12. Pochettino ML, Martínez MR, Arenas PM, Crivos M, Navone G, Digiani C, et al. Fitoterapia Mbyá: análisis y evaluación del tratamiento de las parasitosis. Delpinoa 2003; 45:301-10.

13. Crivos M, Martínez MR, Navone G, Pochettino ML, Arenas PM, Digiani C, et al. Ethnobiology of parasitosis: the case of two-Mbyá Guarani communities in the Province of Misiones, Argentina. In: Stepp JR, Wyndham FS, Zarger RK, editors. Ethnobiology and biocultural diversity. Athens: University of Georgia Press; 2002. p. 258-69.

14. Giraudo AR, Povedano H, Belgrano MJ, Krauczuc ER, Pardiñas U, Miquelarena A, et al. Biodiversity status of the Interior Atlantic Forest of Argentina. In: Galindo-Leal C, Gusmão-Câmara I, editors. The Atlantic Forest of South America: biodiversity status, threats and outlook. Washington DC: Island Press; 2003. p. 160-80.

15. Olson DM, Dinerstein E. The global 2000: a representation approach to conserving the earth's most biologically valuable region. Conserv Biol 1998; 12:502-15.

16. Martínez MR, Crivos M, Remorini C. Etnografía de la vejez en comunidades Mbyá-Guaraní, provincia de Misiones, Argentina. In: Guerci A, Consiglieri S, editors. Biblioteca di antropologia della Salute. Genova: Erga Edizioni; 2002. p. 206-22.

17. Pochettino MI, Martínez MR, Crivos M. Landscape domestication among two Mbyá-Guaraní communities in Misiones, Argentina. In: Stepp JR, Wyndham FS, Zarger RK, editors. Proceedings of the $7^{\text {th }}$ International Congress of Ethnobiology: Ethnobiology and Biocultural Diversity. Athnes: International Society of Ethnobiology/University of Georgia Press; 2002. p. 696-705.

18. Feldman RE, Guardis MV. Diagnóstico coproparasitológico. Fundamentos, normas, metodología, bioseguridad, control de calidad. Nueva guía práctica. Revista de la Federación Bioquímica de la Provincia de Buenos Aires, La Plata 1990; Suppl:165.

19. World Health Organization. Basic laboratory methods in medical parasitology. Geneva: World Health Organization; 1991.

20. Morales G, Pino L. Parasitología cuantitativa. Caracas: Editora Acta Científica Venezolana; 1987.

21. Lohman TG, Roche AF, Martorell KM. Anthropometric standardization reference manual. Champaign: Human Kinetics Books; 1998.

22. Frisancho AR. Anthropometric standards for the assessment of growth and nutritional status. Ann Arbor: University of Michigan Press; 1990.

23. Gorstein J, Sullivan K, Yip R, De Onis M, Trowbridge $\mathrm{F}$, Fajans $\mathrm{P}$, et al. Issues in the assessment of nutritional status using anthropometry. Bull World Health Organ 1994; 72:273-83.

24. Miranda RA, Xavier FB, Menezes RC. Parasitismo intestinal em uma aldeia indígena Parakaná, sudeste do Estado do Pará, Brasil. Cad Saúde Pública $1998 ; 14: 507-11$.

25. Fontbonne A, Freese-de-Carvalho E, Acioli MD, Sá GA, Cesse EAP. Fatores de risco para poliparasitismo intestinal em uma comunidade indígena de Pernambuco, Brasil. Cad Saúde Pública 2001, 17:367-73.

26. Pawlowski ZS, Schad GA, Stott GJ. Infección y anemia por anquilostomas. Geneva: Organización Mundial de la Salud; 1992.

27. Ferrari JO, Farreira MU, Camargo LMA, Ferreira CS. Intestinal parasites among Karitiana indians from Rondônia State, Brazil. Rev Inst Med Trop São Paulo 1992; 34:223-5.

28. Miranda RA, Xavier FB, Menezes RC. Parasitismo intestinal e desnutrição proteico-energética em crianças menores de 10 anos de idade de duas aldeias indígenas na tribo Parakaná, sudeste do Estado do Pará - Brasil. Arq Bras Pediatr 1997; 4:169-73. 
29. Miranda RA, Xavier FB, Nascimento JR, Menezes RC. Prevalência de parasitismo intestinal nas aldeias indígenas da tribo Tembe, Amazônia Oriental Brasileira. Rev Soc Bras Med Trop 1999; 32: 389-93.

30. Laurence DN, Neel JV, Abadie SH, Moore LL, Adams LJ, Healy GR, et al. Estudos epidemiologicos entre populaçoes Amerindias da Amazonia. III. Parasitoses intestinais em povoaçoes recentemente contactadas e em aculturaçao. Acta Amaz 1983; 13:393-407.

31. Tsuyuoka R, Bailey JW, Guimarães AM, Gurgel RQ, Cuevas LE. Anemia and intestinal parasitic infections in primary school students in Aracaju, Sergipe, Brazil. Cad Saude Publica 1999; 15:413-21.

32. Atukorala TM, Lanerolle P. Soil transmitted helminthic infection and its effect on nutritional status of adolescent schoolgirls of low socioeconomic status in Sri Lanka. J Trop Ped 1999; 45:1822.

33. Moore SR, Lima AA, Conaway MR, Schorling JB, Soares AM, Guerrant RL. Early childhood diarrhoea and helminthiases associate with longterm linear growth faltering. Int J Epidemiol 2001; 30:1457-64.
34. Oyhenart EE, Techenski MF, Orden BA. Nutritional status in two Mbyá-Guaraní communities from Misiones (Argentina). Homo 2004; 54:170-9.

35. Santos RV, Coimbra Jr. CEA. Socieconomic transition and physical growth of Tupi-Monde Amerindian children of the Aripuana Park, Brazilian Amazon. Hum Biol 1991; 63:795-819.

36. Orr CM, Dufour DL, Patton JQ. A comparison of anthropometric indices of nutritional status in Tukanoan and Achuar Amerindians. Am J Hum Biol 2001; 13:301-9.

37. Mattos A, Morais MB, Rodríguez DA, Baruzzi R. Nutritional status and dietary habits of Indian children from Alto Xingú (Central Brazil) according to age. J Am Coll Nutr 1999; 18:88-94.

38. Santos RV. Crescimento físico e estado nutricional de populações indígenas brasileiras. Cad Saúde Pública 1993; 9:46-57.

39. Friedlaender JS. The Solomon Islands Project: a long term study of health, human biology and culture change. Oxford: Clarendon Press; 1987.

Recibido el 04/Mar/2005

Versión final presentada el 25/Jul/2005

Aprobado el 06/Sep/2005 